\title{
SIGAA Mobile - $O$ caso de sucesso da ferramenta de gestão acadêmica na era da computação móvel
}

\author{
Itamir Barroca Filho', Gibeon Aquino², José Guilherme Santa Rosa ${ }^{3}$ \\ ${ }^{1}$ Superintendência de Informática - Universidade Federal do Rio Grande do Norte \\ (UFRN) \\ ${ }^{2}$ Departamento de Informática e Matemática Aplicada - Universidade Federal do Rio \\ Grande do Norte (UFRN) \\ ${ }^{3}$ Departamento de Artes - Universidade Federal do Rio Grande do Norte (UFRN) \\ itamir@info.ufrn.br, gibeonedimap.ufrn.br, santarosaecchla.ufrn.br
}

\begin{abstract}
With the advent of mobile devices a new usage scenario of computing is emerging, changing old habits and creating new forms for society access information and interact with computer systems. This scenario requires new types of applications with requirements, specific capabilities and limitations. For this reason, the development of information systems to this new context requires that we review the current knowledge we have about the software development to fit into it. In this article we present successful strategies used to develop a mobile application from a existing web information system.
\end{abstract}

Resumo. Com o advento dos dispositivos móveis um novo cenário de uso da computação está surgindo, mudando os velhos hábitos e criando novas formas da sociedade acessar informações $e$ interagir com os sistemas computacionais. Este cenário, com requisitos, capacidades e limitações específicas, exige novos tipos de aplicações. Por esta razão, o desenvolvimento de sistemas de informações para esse novo contexto exige que revisemos o conhecimento atual que possuímos sobre desenvolvimento de software para nos ajustarmos ao mesmo. Neste artigo serão apresentadas estratégias de sucesso utilizadas para implementar uma aplicação para dispositivos móveis a partir de um sistema de gestão acadêmica web existente.

\section{Introdução}

A computação móvel está cada dia mais presente no cotidiano das pessoas. Nos smartphones e tablets atuais temos poder de processamento que até um tempo atrás só existia em computadores "modernos", com grande capacidade de memória e processamento. De acordo com o Gartner, 1,75 bilhões de pessoas possuem smartphones com capacidades avançadas [Gartner 2013]. Dessa forma, a informação passou a ser acessível a partir de dispositivos móveis poderosos em termos de recursos e com tamanhos cada vez menores. Além disso, como consequência da diversidade de recursos e possibilidades oferecidos por estes dispositivos tem-se observado um aumento muito grande nas vendas dos mesmos nos últimos anos. A International Data 
Corporation (IDC) estima que a venda global de smartphones deva crescer $32,7 \%$ em 2013, chegando a 958,8 milhões de unidades vendidas nesse ano [IDC 2013].

Como resultado da ascendência das vendas dos dispositivos, cresce também a demanda por novas aplicações. Isto pode ser observado pelo crescente número de downloads de aplicativos nos mercados de aplicações mobile, como por exemplo, o Google Play e Apple App Store [IDC 2010]. Dessa forma, percebemos que os sistemas de informação tradicionais estão passando um processo de adaptação para se adequar a essa nova forma de acesso, que está sendo possibilitada pelos dispositivos móveis atuais. Entretanto, é importante destacar que a criação dessas aplicações envolvem atividades tais como: o desenvolvimento da aplicação mobile considerando a plataforma destino (Android, iOS, Web Mobile); integração com serviços exclusivos nesses dispositivos, com por exemplo GPS, SMS e NFC; desenvolvimento/evolução do sistemas de informação web existente; e a integração entre as aplicações móveis e esses sistemas web. Não podemos deixar de considerar também as restrições que essa transformação deve considerar, como por exemplo, tamanho de tela e conectividade dos dispositivos móveis.

Esse artigo tem o objetivo de relatar a experiência da criação de uma aplicação para plataforma mobile no contexto de um sistema de informação web existente na Universidade Federal do Rio Grande do Norte (UFRN) denominado Sistema Integrado de Gestão de Atividades Acadêmicas (SIGAA). O objetivo dessa aplicação mobile é prover novas formas de interação dos usuários com as informações do SIGAA, que será apresentado na seção 2, a partir de dispositivos móveis. Na seção 3, será apresentada a metodologia para adaptação do SIGAA e criação do SIGAA mobile. O SIGAA mobile e os resultados obtidos com publicação no mercado de aplicativos móveis do mesmo serão apresentados respectivamente nas seções 4 e 5. Por fim, na seção 6 serão apresentadas as considerações finais sobre esse artigo.

\section{SIGAA: Sistema Integrado de Gestão de Atividades Acadêmicas}

O SIGAA é um sistema de informação web corporativo que informatiza os procedimentos da área acadêmica através dos módulos de: graduação, pós-graduação (stricto e lato sensu), ensino técnico, ensino médio e infantil, submissão e controle de projetos e bolsistas de pesquisa, submissão e controle de ações de extensão, submissão e controle dos projetos de ensino (monitoria e inovações), registro e relatórios da produção acadêmica dos docentes, atividades de ensino a distância e um ambiente virtual de aprendizado denominado Turma Virtual. A Turma Virtual é o "local" no qual todas as informações de uma determinada turma são acessadas e gerenciadas, e tem como o objetivo principal aumentar a interação entre alunos e professores no processo de ensino-aprendizagem. Entre as funcionalidades oferecidas destacam-se: Plano De Curso, Participantes e Programa do Curso; Fóruns, Chat Da Turma, Vídeo Chat ao Vivo; Notícias, Frequência e Notas; Material Didático (Sites, Vídeos, Arquivos), Enquetes, Tarefas e Questionários. Vale ressaltar que as funcionalidades são acessíveis por docentes e discentes, que possuem nível de acesso diferente dependendo da operação.

Além da Turma Virtual, o SIGAA possuem muitos outros módulos, apresentados na Figura 1 e são habilitados de acordo com os perfis dos usuários. 
Atualmente o SIGAA possui 41.397 usuários divididos entre perfis de estudantes, professores e técnicos. Esse sistema foi desenvolvido utilizando tecnologias livres como: Java, Hibernate, JavaServer Faces, Richfaces, Struts, EJB e Spring. Utiliza PostgreSQL como SGBD e disponibilizado através do servidor de aplicação JBoss. Em termos de métricas de software, o SIGAA possui 646.382 linhas de código, 4750 classes e 1.135 tabelas contidas em 40 esquemas. Com relação às métricas funcionais, contém 1.858 funcionalidades, contabilizando um total de 22.369 pontos de função (contagem baseada no método NESMA).

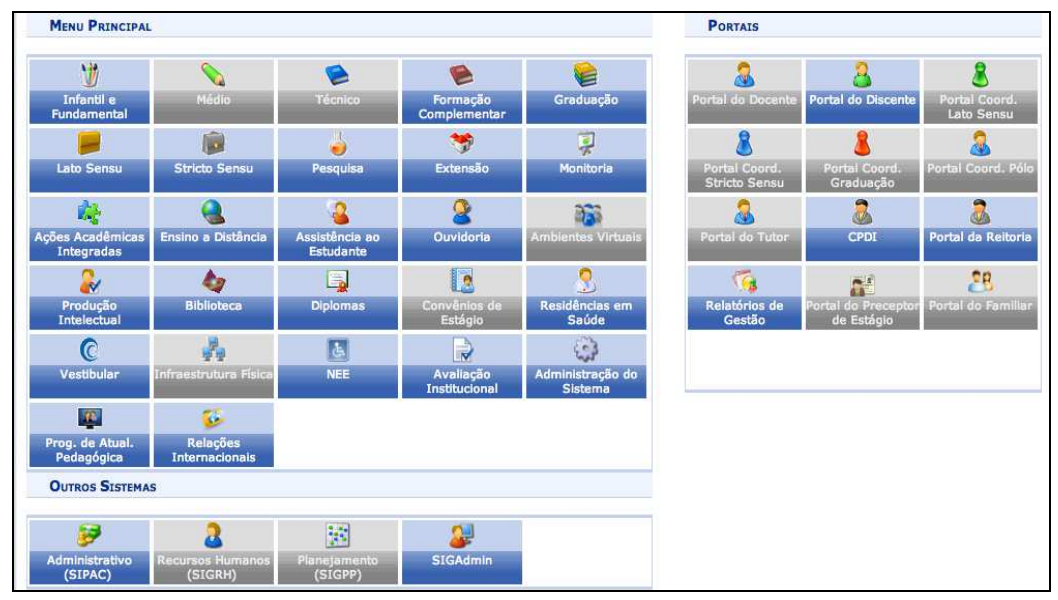

Figura 1. Módulos funcionais do SIGAA.

Com o sucesso do SIGAA na gestão de atividades acadêmicas, em 2009 o mesmo foi disponibilizado para outras universidades federais brasileiras. Atualmente aproximadamente dezenove universidades federais estão utilizando o SIGAA.

\section{Metodologia para adaptação do sistema}

Conforme descrito na introdução, o desenvolvimento de aplicações mobile envolve um contexto específico e requer novas abordagens relacionadas as diferentes facetas do desenvolvimento de software. Nesta seção apresentaremos a categorização da metodologia para adaptação do SIGAA em três grupos: (a) Negócio, que envolve estratégias relacionadas ao escopo, envolvimento das partes interessadas e publicidade; (b) Aspectos técnicos, que envolve estratégias relacionadas ao código fonte do produto, como soluções arquiteturais, utilização de tecnologias, frameworks, padrões de projetos e boas práticas; (c) Interface gráfica, que envolve estratégias relacionadas à aparência visual e a forma como os usuários interagem com a aplicação mobile.

\subsection{Estratégias relacionadas ao negócio}

No início do projeto, é muito importante planejar quais funcionalidades são relevantes para o contexto mobile. É importante destacar que o processo de criação de uma aplicação mobile a partir de um sistema de informação web não é um mapeamento direto de funcionalidade para funcionalidade. Esse tipo de simplificação é um erro comum e deve ser tratado com cuidado. Dispositivos móveis possuem restrições específicas como tamanho de tela, dificuldade de digitação de longos textos e não há garantia disponibilidade de acesso à rede. Além disso, possui formas diferentes de interação com suporte a touch screen, gestos e ações rápidas. Por essa razão é 
importante seguir algumas estratégias especificas nos projetos que envolvem desenvolvimento de aplicações mobile.

Uma dessas estratégias está relacionada ao escopo ou mais especificamente a escolha das funcionalidades e a forma como as mesmas precisam ser adaptadas para esse novo contexto. Então, essa estratégia é subdivida em quatro práticas: (i) escolher funcionalidades populares; (ii) evitar funcionalidades de longos passos ou longos formulários; (iii) adaptar funcionalidades existentes; (iv) criar funcionalidades específicas para o contexto mobile. Podemos perceber, conforme descrito na seção 2, que o SIGAA possui muitas funcionalidades e não faz sentido programar cada uma dessas funcionalidades no SIGAA mobile. Por essa razão minimizamos o escopo, baseando-se nos requisitos funcionais do SIGAA, mas apenas implementando as operações mais populares e convenientes (práticas i e ii). Além disso, algumas funcionalidades precisaram ser revisadas e adaptadas considerando as restrições desse novo contexto, conforme sugerido pela prática iii. A primeira versão do SIGAA mobile publicada em abril de 2012, possuía apenas treze funcionalidades e suportava apenas os perfis de professor e estudantes. Já a versão publicada em março de 2013 possuía novas funcionalidades que não existiam no SIGAA web, mas que eram importantes para o contexto mobile. Procurou-se dar prioridade às funcionalidades relacionadas à interação entre docentes e discentes no processo de ensino de aprendizagem, por isso maior parte das operações atualmente implementadas são relativas à Turma Virtual.

Outra estratégia importante é o envolvimento dos usuários durante a fase de desenvolvimento. Para uma iniciativa de sucesso no desenvolvimento de software é importante o envolvimento do usuário [Beck e Andres 2004], [Majid et al 2010], e no desenvolvimento voltado para o contexto mobile essa prática se torna ainda mais importante. Os potenciais usuários da versão mobile já utilizam o SIGAA web, e por esse motivo esperam que essa aplicação seja tão boa como àquela que já existe. Além disso, eles esperam que essa aplicação mobile torne o trabalho de usar o sistema mais fácil quando eles estiverem em um dispositivo móvel. Com base nessa estratégia, tentamos envolver vários professores e alunos do projeto antes de iniciar desenvolvimento, a fim de receber as suas expectativas e feedbacks sobre nossos planos.

Por fim, uma das estratégias mais importantes é a publicidade em torno da aplicação mobile. O potencial usuário deve saber essa nova forma de interação com as informações do sistema de informação web e se sentirem motivados para experimentar esta nova maneira de acessar o sistema. Apenas publicar o aplicativo em uma loja de plataforma, como por exemplo: Google Play ou Apple App Store, não é suficiente para torná-lo conhecido entre seus potenciais usuários. Por este motivo, a sua existência deve ser bem divulgada para o público-alvo. No nosso caso, na primeira versão do SIGAA mobile não ocorreu nenhuma ação para comunicar a disponibilidade do mesmo para seus usuários. Entretanto, na segunda versão disponibilizada, a AGECOM (Agencia de Comunicação da UFRN) fez um trabalho de divulgação dessa aplicação e o número de downloads aumentou significativamente.

\subsection{Estratégias relacionadas aos aspectos técnicos}

O primeiro desafio que precisa ser considerado é como realizar a integração da aplicação mobile com o sistema de informação existente. Além disso, precisamos 
analisar como reutilizar os componentes da camada de negócio já desenvolvidos no sistema de informação existente. Atualmente, a utilização do padrão de desenvolvimento em camadas [Buschmann 1996] é comum em especial para os sistemas de informações web. Por esta razão, uma abordagem genérica de que podem ser utilizada para integrar a aplicação mobile com o sistema web existente é a criação de uma nova camada, para fornecer um conjunto de serviços que deve ser utilizada pelo aplicativo mobile. Essa nova camada permite a integração com a camada de regras de negócio já existente e estável no sistema web, conforme apresentado na Figura 2. No projeto SIGAA mobile, esta camada é composta por vários Web Services RESTful que usa os componentes de negócios (session beans, entidades persistentes e classes utilitárias) fornecidos pela camada de negócio existente no SIGAA web.

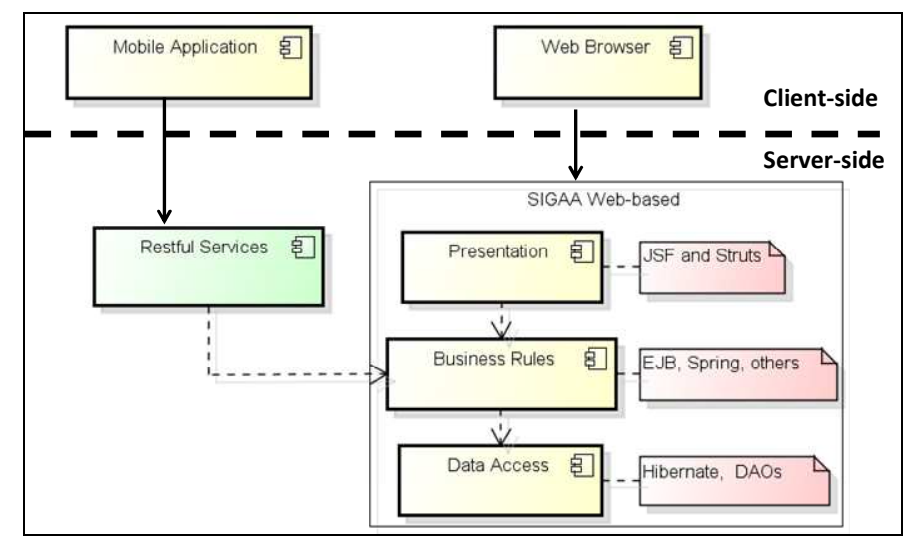

Figura 2. Solução baseada na criação de uma nova camada de services para integrar o SIGAA com o SIGAA mobile.

Por fim, um das características técnicas mais importantes nesse novo contexto diz respeito à disponibilidade da conectividade com a Internet. Os dispositivos móveis se conectam com a Internet através de conexões sem fio e a disponibilidade das mesmas depende principalmente da localização geográfica onde o usuário se encontra. Além disso, a conectividade pode se apresentar de maneira instável, enquanto o usuário está interagindo com o aplicativo mobile. Por este motivo, uma das funções mais interessantes implementada no SIGAA mobile foi a capacidade de funcionar off-line. Mesmo quando não há conectividade com a Internet, o usuário pode entrar no local e fazer todas as operações que ele pode fazer quando ele está on-line. Isso é possível pois o SIGAA mobile mantém um cache de dados local quando o usuário está off-line e sincroniza essa informação com o SIGAA web, quando o mesmo volta a estar on-line. Também programamos um mecanismo que detecta a indisponibilidade de conectividade com a Internet a qualquer momento enquanto o usuário está interagindo com o SIGAA mobile. Caso a conectividade seja perdida, o mesmo pergunta ao usuário se ele quer continuar seu trabalho em modo off-line, armazenando as entradas de dados do usuário em um banco de dados local e mais tarde, quando a conectividade é retomada essas informações são sincronizadas.

\subsection{Estratégias relacionadas à interface com usuário}

As aplicações móveis tendem a fornecer vantagens relevantes para seus usuários em termos de design e usabilidade [Nayebi 2012]. Por esta razão, exige novas estratégias de interação e identidade visual própria com o intuito de diferir da versão móvel do sistema 
web. Uma abordagem perigosa é tentar imitar o sistema web existente. Aplicativos famosos como, por exemplo, o Facebook e Twitter possuem suas próprias aparências e usabilidade em sua versão mobile. Portanto, uma estratégia importante é a pensar na aplicação móvel como algo novo, voltado para um dispositivo móvel, mesmo nascendo a partir de um sistema web e, principalmente, elaborar o projeto da interface a partir da identificação das necessidades, habilidades e restrições dos usuários. Para tanto, a equipe de pesquisa e desenvolvimento da interface adotou a abordagem do Design Participativo - na qual os usuários (no caso do SIGAA, alunos e professores) são envolvidos e participam ativamente em todas as etapas do projeto [Muller 1999], [Santa Rosa \& Moraes 2012]. Para tanto, foram realizadas atividades participativas com alunos e professores de diversos cursos da UFRN, tais como: grupo focal, avaliação, avaliação cooperativa e testes de usabilidade.

De acordo com Nielsen (2003), usabilidade é a capacidade de um produto ou sistema, em termos funcionais-humanos, de ser usado com facilidade e eficácia por um segmento específico de usuários. Destaca-se, que a ISO 9241-11 estabelece que a usabilidade refere-se à eficiência e à satisfação com as quais usuários específicos atingem objetivos específicos em determinados contextos de uso [ISO 1993], o que no caso do SIGAA Mobile, é extremamente pertinente, posto que alunos e professores utilizaram o sistema SIGAA por meio de dispositivos móveis, em contextos que não utilizariam se estivessem acessando o sistema por meio do Desktop ou, mesmo, notebook.

Com base nesse entendimento, no SIGAA mobile, primeiro foi definido um logotipo para ser utilizado como um ícone da aplicação. Posteriormente cada funcionalidade foi avaliada em termos de passos, a quantidade e disposição dos campos dos formulários. Por fim, as ações mais importantes foram a avaliação de usabilidade e a proposição de recomendações relacionadas a aspectos ergonômicos e de usabilidade. A realização de sessões de grupo focal com alunos de diferentes cursos e a avaliação cooperativa com professores, permitiu à equipe de design identificar não só os principais e novos contextos de uso e objetivos como, também, compreender as necessidades dos usuários (no caso sujeitos da aprendizagem - utilizadores do Sistema de Gerenciamento Acadêmico). Com base nos resultados dos testes de usabilidade, triangulados com os resultados das técnicas de grupo focal e avaliação cooperativa, foram propostas recomendações para a elaboração da interface.

Dentre as principais recomendações ergonômicas e de usabilidade propostas pela equipe de design à equipe de desenvolvimento do sistema citam-se: a) utilizar interface com design minimalista; b) sempre considerar o uso de linguagem natural ao usuário para a apresentação das informações especialmente nas datas e em informações de horários das aulas; c) considerar grid com espaçamento suficiente para criar áreas em branco e separadores de conteúdos, facilitando a seleção de itens e melhorando a interação; d) apresentar opções mais acessadas acima da altura de corte da tela; e) optar pela interação por tap. Nos locais onde o slide da tela é necessário para a interação, é preciso sinalizar estas opção de maneira clara e legível.

Após a realização da avaliação de usabilidade a equipe de design considerou pertinente a realização de um estudo a respeito do modelo de interação do módulo de lançamento de frequência. Para tanto, foi aplicada a técnica de prototipagem de alta 
definição e, posteriormente aplicado um questionário de satisfação entre os professores da instituição, sujeitos da pesquisa. Para esta avaliação foram consideradas três propostas de solução, também denominadas de modelos de interação [Santa Rosa, Gurgel e Passos 2012].

Participaram dos Testes de Usabilidade da Prototipagem de Alta Definição docentes da área de Ciências e Tecnologia $(n=3)$ e de Design $(n=2)$. Foi solicitado que os participantes mudassem os status de frequência de três alunos em cada modelo e expressasse livremente suas impressões. Logo após os testes foi aplicado questionário adaptado do modelo QUIS (Questionnaire for User Interface Satisfaction). De acordo com os resultados foi possível verificar que, embora o modelo II tivesse sido considerado mais adequado pelos professores, sujeitos da pesquisa, o modelo III, foi considerado o mais amigável, por permitir uma seleção da frequência de modo mais rápido e agradável. Destaca-se que este fator deve ser levado em consideração, principalmente, nos casos em que o professor administra as frequências e faltas em turmas numerosas - como é o caso do curso de Ciência \& Tecnologia.

\section{SIGAA Mobile}

O SIGAA mobile foi desenvolvido para plataforma Android com a utilização das estratégias descritas na seção 3 e suas camadas são apresentadas na Figura 3. A camada View contém todas as activities desse aplicativo. Essas activities são componentes que interagem diretamente com a interface gráfica e manipula os eventos do usuário. Essa camada utilizada a camada denominada Bussiness Delegate que responsável por delegar a utilização da camada de cache local de dados denominada Cache Data Access, ou a saída da informação pela camada de comunicação denominada Communication Channel, baseada na disponibilidade de conexão do usuário com a Internet.

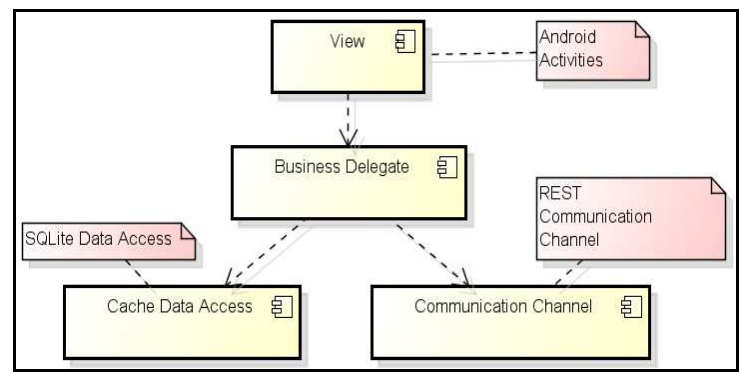

Figura 3. Arquitetura de software do SIGAA mobile.

Dentre as funcionalidades disponibilizadas por essa aplicação mobile tem-se para o aluno no menu principal, apresentada na Figura 4, a possibilidade consultar as turmas que o mesmo se matriculou; emissão de documentos como histórico, atestado de matricula e declaração de vínculo; visualizar os horários e as notas do semestre e ir para a versão web. Ao selecionar uma turma, o aluno poderá ter informações sobre sua frequência, suas notas, as notícias disponibilizadas pelo professor, visualizar e contatar os demais participantes da turma e ter acesso aos tópicos de aulas, podendo, por exemplo, baixar arquivos enviados pelos professores. Para o perfil do professor, o SIGAA Mobile possibilita informações de turmas, alunos e o lançamento da frequência dos mesmos. 


\section{Resultados do novo produto}

Nesta seção vamos apresentar algumas estatísticas sobre o uso do SIGAA mobile. Com o Android Developer Console extraímos informações sobre downloads considerando as instalações ativas. Atualmente, o SIGAA mobile está instalado em cerca de 3.510 dispositivos (instalações ativas) e com uma avaliação dos usuários de 4.7 (de um máximo de 5). O crescimento desde a sua disponibilização é apresentado na Figura 5. Ele foi enviado para o Google Play em 25/04/2012 e o número de download está a aumentando desde então.

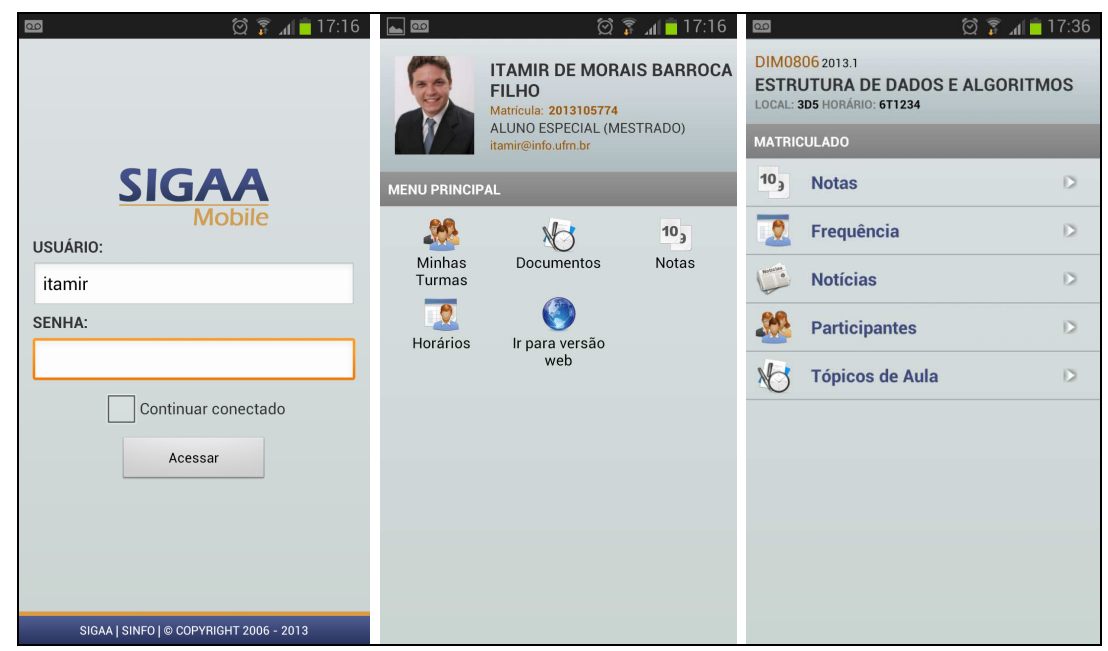

Figura 4. Telas do SIGAA mobile.

Depois da obtenção das informações de downloads, utilizamos a infra-estrutura logs SIGAA para descobrir o número de acessos diários e funcionalidades mais utilizadas do SIGAA mobile. O SIGAA tem um registo de base de dados controlada por um processo de assíncrono persistindo informações sobre todas as operações do usuário.

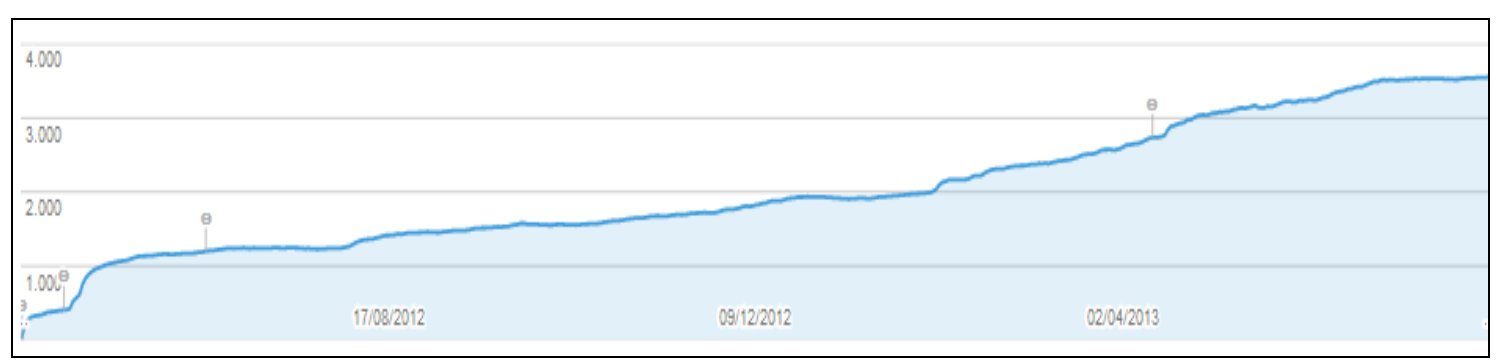

Figura 5. Quantidade de instalações ativas do SIGAA Mobile.

Analisando logins de acesso diárias no SIGAA mobile, descobrimos que desde o seu lançamento, esta aplicação tem 124.643 logins e uma média, cerca de 410 logins diárias. Notamos o aumento nos números no início e no final do académico período de actividade, tal como apresentado no gráfico a Figura 6. Para exemplo, o mês de férias, o número de acesso diminui, mas imediatamente antes e imediatamente após o número de acesso aumenta. 


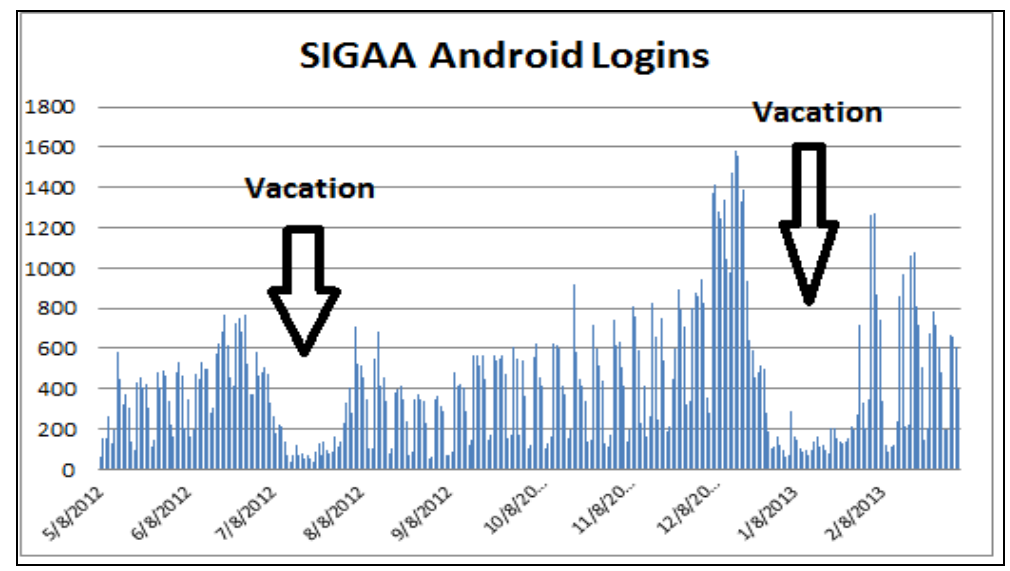

Figura 6. Frequência de logins no SIGAA mobile.

\section{Considerações Finais}

Esse artigo apresentou a experiência da criação do SIGAA mobile no contexto de um sistema de informação web de gestão acadêmica, o SIGAA. Através dessa experiência foi possível identificar estratégias de sucesso relacionadas ao negócio, aspectos técnicos e interface gráfica, que se mostram úteis para criação de aplicativos móveis, particularmente na área de gestão acadêmica e envolvendo sistemas de informações web pré-existentes.

Como trabalhos de programação futuros, planejamos o desenvolvimento de novas funcionalidades para o SIGAA mobile, tais como: compartilhamento de arquivos diretamente na turma virtual a partir do dispositivo; recebimento de notificações de novidades (mensagens, publicações de notas e tarefas); recebimento de notificações de alertas/lembretes (datas de provas, prazo de trabalhos/tarefas e aproximação do limite de faltas) e comunicação em grupo possibilitando a interação entre os alunos e participantes. Atualmente algumas dessas funcionalidades já estão sendo desenvolvidas.

Com relação às estratégias, acreditamos que embora este trabalho relate a experiência específica do projeto SIGAA mobile, as mesmas podem ser generalizadas e aplicadas em outros projetos semelhantes. Especificamente em projetos de desenvolvimento de versões mobile de sistemas de informação web já existentes. No entanto, para ser mais confiante da eficácia dessas estratégias é necessária a realização de um estudo aprofundado da aplicabilidade das mesmas. Por esta razão, iremos utilizalas em outros projetos semelhantes, com o objetivo de recolher mais evidências sobre o tema.

Destaca-se que a abordagem do Design Participativo, além de contribuir para o levantamento de necessidades e requisitos de usabilidade, propiciou a todos os envolvidos no projeto (designers, engenheiros da computação, analistas de sistemas e programadores) melhor compreensão a respeito dos utilizadores do sistema - no caso, os sujeitos da aprendizagem - criando condições necessárias para que o sistema seja desenvolvido com base nos preceitos do Design Centrado no Usuário.

Cabe enfatizar, ainda, que ao considerar os usuários (sujeitos da aprendizagem) em todas as etapas do projeto e desenvolvimento do sistema vislumbra-se a criação de um sistema de gerenciamento de informações acadêmicas considerando que a maior 
parte da carga cognitiva destinada a sua manipulação (carga extrínseca) deve ser minimizada, de modo que os alunos e professores possam se dedicar as atividades inerentes à relação ensino-aprendizagem.

Por fim, destaca-se que, por meio das diversas técnicas de avaliação da usabilidade, verificou-se a importância de implementar funções tais como: identificação e localização de materiais bibliográficos na biblioteca, localização por GPS de setores, departamentos e salas de aula e, principalmente, de recursos que propiciem maior interação entre alunos e professores, de modo que o sistema possa oferecer contribuições para uma relação de ensino-aprendizagem centrada no aluno e de acordo com os preceitos da teoria de aprendizagem construtivista.

\section{Agradecimentos}

Ao Andrei Gurgel (aluno de mestrado do Programa de Pós-Graduação em Design, UFRN) e ao Marcel Passos (bolsista e aluno do Curso de Bacharelado em Design, UFRN).

À Superintendência de Informática da Universidade Federal do Rio Grande do Norte e ao Laboratório de Ergodesign de Interfaces e Usabilidade (LEXUS/UFRN).

\section{Referências}

Beck K.; Andres, C. Extreme Programming Explained: Embrace Change (2nd Edition). 2004. Addison-Wesley Professional.

Buschmann, F.; Meunier, R.; Rohnert, H.; Sommerlad, P.; Stal, M. (1996) PatternOriented Software Architecture: A System of Patterns. John Wiley \& Sons, Inc., New York, NY, USA.

Gartner, Inc. (2013) Gartner Says Worldwide Mobile Phone Sales Declined 1.7 Percent in 2012. Egham, UK: GARTNER. Disponível em: http://www.gartner.com/newsroom/id/2335616. Acesso em 20/04/2013.

IDC, Market Analysis. (2010) IDC Forecasts Worldwide Mobile Applications Revenues to Experience More Than 60\% Compound Annual Growth Through 2014. Framinghan, USA: IDC. Disponível em http://www.idc.com/getdoc.jsp?containerId=prUS24143513 Acesso em: 03/07/2013.

IDC, Market Analysis.(2013) Smartphones Expected to Grow 32.7\% in 2013 Fueled By Declining Prices and Strong Emerging Market Demand, According to IDC. Framinghan, USA: IDC. Disponível em http://www.idc.com/getdoc.jsp?containerId=prUS24143513. Acesso em: 03/07/2013.

Majid, R.A.; Noor, N.L.M.; Adnan, W.A.W.; Mansor, S. (2010) A survey on user involvement in software Development Life Cycle from practitioner's perspectives. Computer Sciences and Convergence Information Technology (ICCIT), 2010 5th International Conference on, vol., no., pp.240,243, Nov. 30 2010-Dec. 22010.

Muller, M. et al. Participatory partices in the software lifeycle. In: Handbook of HumanComputer Interactions, 2ed. Amsterdam: Elservier Science B V. 1997. 
Nayebi, F.; Desharnais, J.-M.; Abran, A. (2012) The state of the art of mobile application usability evaluation. Electrical \& Computer Engineering (CCECE), 25th IEEE Canadian Conference on , vol., no., pp.1,4, April 29 2012-May 22012.

Nielsen, J. Usability Engineering. Boston: Academic, Press, 1993.

Santa Rosa, J.G. \& Moraes, A. Design Participativo - técnicas para inclusão de usuários no processo de ergodesign de interfaces. Rio de Janeiro: Rio Books, 2012.

Santa Rosa, J.G., Gurgel, A. e Passos, M. Técnicas Baseadas em Etnografia e Prototipagem no Design de interface de Aplicativo Mobile para Gerenciamento Acadêmico. InfoDesign. São Paulo: v. 9 | n. 2 [2012], p. 88 - 99. 